\title{
NEAR-WALL DETERMINATION OF THE TURBULENT PRANDTL NUMBER BASED ON EXPERIMENTS, NUMERICAL SIMULATION AND ANALYTICAL MODELS
}

\author{
Steiner H.*, Irrenfried C. and G. Brenn \\ *Author for correspondence \\ Institute of Fluid Mechanics and Heat Transfer, \\ Graz University of Technology, \\ Austria, \\ E-mail: helfried.steiner@tugraz.at
}

\begin{abstract}
The Reynolds-averaged computation of turbulent flow with heat transfer most commonly introduces the turbulent Prandtl number to relate the turbulent fluxes of momentum and heat. Its significant deviation from a uniform bulk flow value for high molecular Prandtl numbers requires a reliable description of this parameter for predicting accurately the heat transfer. The present study proposes a model for the near-wall variation of this important quantity for use in an analytically computed solution of heated turbulent pipe flow. The comparison of the predictions against results from Direct Numerical Simulation (DNS) and experiments proves the proposed analytical approach as a computationally efficient alternative to the much costlier numerical approach with still acceptable accuracy. The analytically obtained results do not only demonstrate the reliability of the proposed model for the near-wall behavior of the turbulent Prandtl number, but also highlight the significance of the dependence of the material properties on the temperature. Numerical simulations mostly neglect this effect to avoid a further increase of the already high computational costs associated with the discretized solution of the heated/cooled flow field.
\end{abstract}

\section{INTRODUCTION}

The appropriate setting of the turbulent Prandtl number $\operatorname{Pr}_{T}$ as an input into most standard thermal wall boundary conditions used in $R A N S$-type simulations is of major importance of the accuracy of the predictions. While the uniformity of this parameter being close to unity $\left(\operatorname{Pr}_{T}=\operatorname{Pr}_{T, \infty} \approx\right.$ const . $)$ has been proven for the bulk flow region remote from the heated/cooled wall by numerous experimental and computational studies (see, e.g., [1-4]), its near-wall behavior is still unclear, especially in the case of high molecular Prandtl numbers. A couple of models for the near-wall asymptotics of the turbulent Prandtl number have been proposed thus far, e.g., [5-8], but these ansatzes were mostly not validated, neither against well resolved detailed numerical simulations, nor against experiments. The vanishingly thin conductive sub-layer for increasing molecular Prandtl numbers impedes a direct experimental measurement of the turbulent fluctuations of the temperature and velocity, and their respective

\section{NOMENCLATURE}

\begin{tabular}{|c|c|c|}
\hline$A, B$ & {$[-]$} & Model coefficients \\
\hline$c_{p}$ & {$[\mathrm{~J} / \mathrm{kgK}]$} & Specific heat capacity \\
\hline$D$ & {$[\mathrm{~m}]$} & Diameter \\
\hline$h$ & {$[\mathrm{~J} / \mathrm{kgK}]$} & Specific enthalpy \\
\hline$l_{m}$ & {$[\mathrm{~m}]$} & Mixing length \\
\hline$\dot{m}$ & {$[\mathrm{~kg} / \mathrm{s}]$} & Massflow rate \\
\hline$N u$ & {$[-]$} & Nusselt number \\
\hline$p$ & {$[\mathrm{~Pa}]$} & Pressure \\
\hline $\operatorname{Pr}$ & {$[-]$} & Prandtl number \\
\hline $\operatorname{Pr}_{T}$ & {$[-]$} & Turbulent Prandtl number \\
\hline $\operatorname{Pr}_{T, \infty}$ & {$[-]$} & Turbulent Prandtl number for $y^{+} \rightarrow \infty$ \\
\hline$q_{i}$ & {$\left[W / m^{2}\right]$} & Conductive heat flux component \\
\hline$r$ & {$[m]$} & Radial coordinate \\
\hline $\operatorname{Re}$ & {$[-]$} & Reynolds number \\
\hline $\operatorname{Re}_{\tau}$ & {$[-]$} & Wall friction Reynolds number \\
\hline$T$ & {$[K]$} & Temperature \\
\hline$u, v, w$ & {$[\mathrm{~m} / \mathrm{s}]$} & Radial, azimuthal, axial velocity component \\
\hline
\end{tabular}

\section{Abbreviations \\ RANS [-] Reynolds-averaged Navier-Stokes equations \\ DNS [-] Direct numerical simulation}

$\begin{array}{cll}\text { Special characters } & \\ \alpha & {\left[\mathrm{W} / \mathrm{m}^{2} \mathrm{~K}\right]} & \text { Heat transfer coefficient } \\ \varepsilon_{q} & {\left[\mathrm{~m}^{2} / \mathrm{s}\right]} & \text { Eddy diffusivity } \\ \lambda & {[\mathrm{W} / \mathrm{mK}]} & \text { Thermal conductivity } \\ \mu & {[\mathrm{Pas}]} & \text { Dynamic viscosity } \\ \mu_{T} & {[\mathrm{Pas}]} & \text { Eddy viscosity } \\ \rho & {\left[\mathrm{kg} / \mathrm{m}^{3}\right]} & \text { Density } \\ \tau_{i j} & {\left[\mathrm{~N} / \mathrm{m}^{2}\right]} & \text { Viscous stress component }\end{array}$

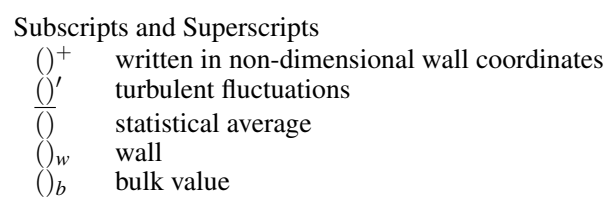

mean gradients, as well as a direct numerical simulation (DNS) due to the exceedingly high computational resolution requirements. The present study proposes a near-wall model for the turbulent Prandtl number based on analytical computations along with observations from Direct Numerical Simulations, which are carried out for molecular Prandtl numbers up to $P r=20$. The analytical predictions produced with this model are compared against experimental data, which were measured on a facility specially designed for investigating heated turbulent pipe flow. This validation was also used for model calibration. 


\section{DIRECT NUMERICAL SIMULATION}

This most accurate approach resolves numerically direct all the physically relevant scales, which restricts its applicability to cases with fairly low Reynolds numbers and molecular Prandtl number not extremely beyond unity, due to the exceedingly high computational coasts. For these cases DNS still produces valuable data for model derivation and evaluation, and is therefore also applied in the presented study. Considering incompressible turbulent pipe flow heated at constant wall heat flux, the present $D N S$ solves numerically directly the full set of the unsteady, three-dimensional conservation equations of mass, momentum and specific enthalpy, written in cylindrical coordinates as

$$
\begin{gathered}
\frac{\partial \rho}{\partial t}+\frac{1}{r} \frac{\partial(r \rho u)}{\partial r}+\frac{1}{r} \frac{\partial \rho v}{\partial \varphi}+\frac{\partial \rho w}{\partial z}=0 \\
\frac{\partial \rho u}{\partial t}+\frac{1}{r} \frac{\partial(r \rho u u)}{\partial r}+\frac{1}{r} \frac{\partial(\rho u v)}{\partial \varphi}-\frac{\rho v^{2}}{r}+\frac{\partial(\rho u w)}{\partial z}= \\
-\frac{\partial p}{\partial r}+\frac{1}{r} \frac{\partial\left(r \tau_{r r}\right)}{\partial r}+\frac{1}{r} \frac{\partial \tau_{r \varphi}}{\partial \varphi}+\frac{\tau_{\varphi \varphi}}{r}+\frac{\partial \tau_{r z}}{\partial z} \\
\frac{\partial \rho v}{\partial t}+\frac{1}{r} \frac{\partial(r \rho v u)}{\partial r}+\frac{1}{r} \frac{\partial(\rho v v)}{\partial \varphi}+\frac{\rho v u}{r}+\frac{\partial(\rho v w)}{\partial z}= \\
-\frac{1}{r} \frac{\partial p}{\partial \varphi}+\frac{1}{r} \frac{\partial\left(r \tau_{\varphi r}\right)}{\partial r}+\frac{\tau_{\varphi r}}{r}+\frac{1}{r} \frac{\partial \tau_{\varphi \varphi}}{\partial \varphi}+\frac{\partial \tau_{\varphi z}}{\partial z} \\
\frac{\partial \rho w}{\partial t}+\frac{1}{r} \frac{\partial(r \rho w u)}{\partial r}+\frac{1}{r} \frac{\partial(\rho w v)}{\partial \varphi}+\frac{\partial(\rho w w)}{\partial z}= \\
-\frac{\partial p}{\partial z}+\frac{1}{r} \frac{\partial\left(r \tau_{z r}\right)}{\partial r}+\frac{1}{r} \frac{\partial \tau_{z \varphi}}{\partial \varphi}+\frac{\partial \tau_{z z}}{\partial z} \\
\frac{\partial \rho h}{\partial t}+\frac{1}{r} \frac{\partial(r \rho u h)}{\partial r}+\frac{1}{r} \frac{\partial(\rho v h)}{\partial \varphi}+\frac{\partial(\rho w h)}{\partial z}= \\
\frac{1}{r} \frac{\partial\left(r q_{r}\right)}{\partial r}+\frac{1}{r} \frac{\partial q_{\varphi}}{\partial \varphi}+\frac{\partial q_{z}}{\partial z}
\end{gathered}
$$

Therein, $u, v$, and $w$ denote the velocities in the radial, azimuthal and axial directions, $r, \varphi$, and $z$, respectively, $p$ is the pressure, $\tau_{i j}$ are the viscous stresses, $h$ is the specific enthalpy, and $q_{i}$ are conductive heat flux components. The density $\rho$ and the material properties were assumed as constant.

The equations were solved using a $4^{\text {th }}$ order accurate FiniteVolume discretization in space and $2^{\text {nd }}$ order accurate AdamsBashforth discretization in time. The computational domain is shown in Figure 1. The smallest cells are located next to the wall with $\Delta r_{\text {min }}^{+}=0.256, R^{+} \Delta \varphi=4.418$ and $\Delta z^{+}=3.516$ measured in wall units $\mu / \rho w_{\tau}$ with the wall friction velocity $w_{\tau}=\left(\tau_{w} / \rho\right)^{(1 / 2)}$. Periodic boundary conditions are used for all dependent variables in the circumferential direction $\varphi$. No-slip boundary conditions are prescribed for the velocities at the wall, and a uniform time averaged wall heat flux and zero temperature fluctuations, implying $\bar{q}=q_{w}$ and $T_{w}-T=0$, are imposed as thermal wall boundary conditions. Considering hydrodynamically and thermally fully developed flow, periodic boundary conditions are imposed for

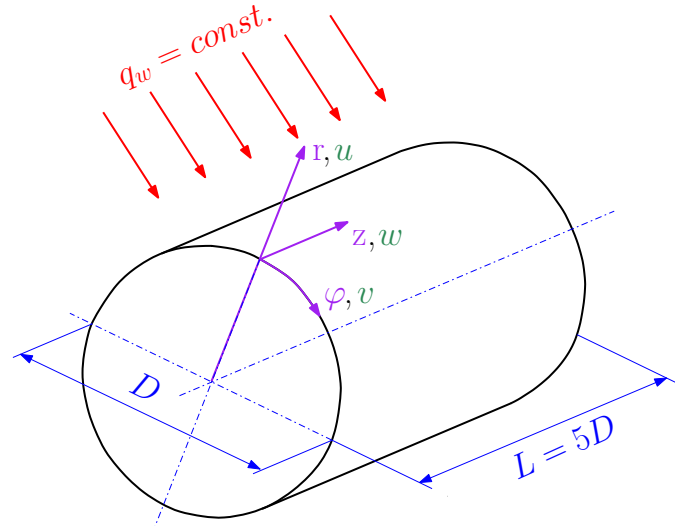

Figure 1. Computational domain

the normalized velocities, pressure fluctuations, and the temperature in the axial direction $z$. The presented DNS considered three cases with $R e_{\tau}=360$, and varying molecular Prandtl numbers $\operatorname{Pr}=1,10$ and 20. With the focus on the modelling of the turbulent Prandtl number, the results were particularly analyzed for this quantity, which is computed from the DNS data as

$$
\operatorname{Pr}_{T}=\frac{\mu_{T}}{\rho \varepsilon_{q}}=\overline{\overline{u^{\prime} w^{\prime}}} \frac{\frac{\partial \bar{T}}{u^{\prime} T^{\prime}}}{\frac{\partial r}{\partial \bar{w}}}
$$

relating by definition the Boussinesq eddy viscosity $\mu_{T}$ to the eddy diffusivity $\varepsilon_{q}$. The primes denote the turbulent fluctuations relative to the statistical averages indicated by the overbars. Figure 2 shows that the turbulent radial Prandtl number remain around unity in the core region and increases with increasing molecular Prandtl number towards the wall. The commonly used assumption of a uniform constant turbulent Prandtl number, often set to $\operatorname{Pr}_{T}=0.85$, evidently does not hold near the wall as the molecular Prandtl number increases. The proposed

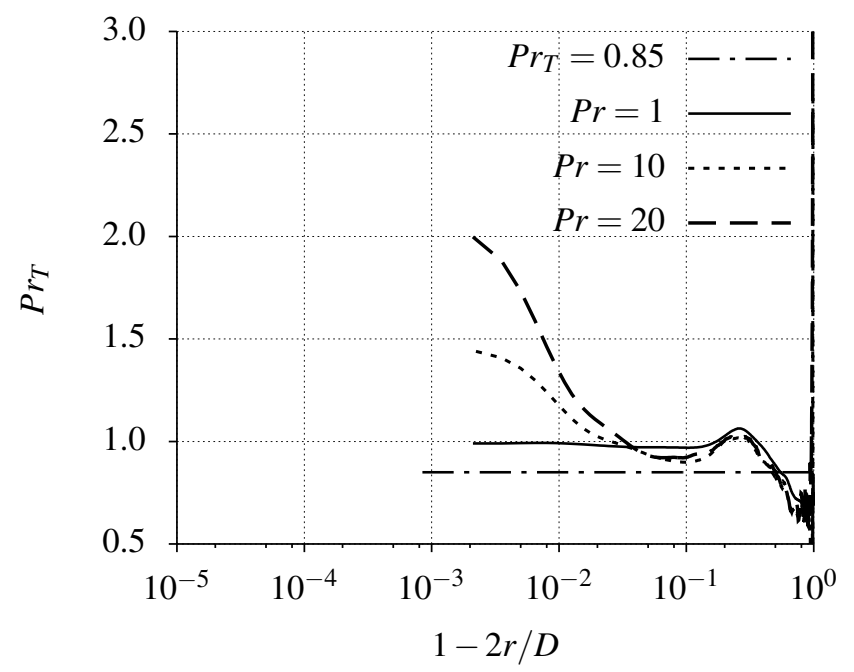

Figure 2. Variation of the turbulent Prandtl number based on the $D N S$ for $\operatorname{Pr}=1 / 10 / 20$ 
model shall particularly reflect this near-wall behavior, as shown further below.

\section{ANALYTICAL MODEL}

The presently shown analytical approach essentially starts out from the work of [9] who analytically considered dynamically and thermally fully developed turbulent pipe flow heated with constant wall heat flux. The fluid is assumed here again as incompressible, but with temperature dependent material properties, such as the dynamic viscosity $\mu$, thermal conductivity $\lambda$ and the specific heat capacity $c_{p}$. Applying these assumptions, the Reynolds-averaged representations of the full set of conservation equations (1)-(5) reduce to

$$
\frac{\partial \bar{p}}{\partial z}=\frac{1}{r} \frac{\partial}{\partial r}\left(r \tau_{t o t}\right)
$$

and

$$
\rho \bar{w} \frac{\partial \bar{h}}{\partial z}=\frac{1}{r} \frac{\partial}{\partial r}\left(r q_{t o t}\right),
$$

The total shear stress $\tau_{\text {tot }}$ and the heat flux $q_{\text {tot }}$ on the right-hand sides of equation (7) and (8), respectively, consists of a laminar and a turbulent contribution

$$
\tau_{t o t}=\mu \frac{\partial \bar{w}}{\partial r}-\rho \overline{u^{\prime} w^{\prime}}=\left(\mu+\mu_{T}\right) \frac{\partial \bar{w}}{\partial r}
$$

and

$$
q_{t o t}=\lambda \frac{\partial \bar{T}}{\partial r}+\rho \overline{u^{\prime} h^{\prime}}=\left(\frac{\lambda}{c_{p}}+\rho \varepsilon_{q}\right) \frac{\partial \bar{h}}{\partial r}
$$

where the turbulent components are modelled here using the Boussinesq eddy viscosity $\mu_{T}$ and an eddy diffusivity $\varepsilon_{q}$ as turbulent transport coefficients.

Given the dynamically and thermally fully developped flow conditions, the axial derivatives on the left-hand side of equation (7) and (8) can be assumed constant across the pipe cross section. As a consequence, both equations can be radially integrated from the center, $r=0$, to the wall, $r=D / 2$, in order to express these axial derivates in terms of the wall fluxes $\tau_{w}$ and $q_{w}$, respectively, such that equation (7) and (8) can be rewritten as

$$
\frac{d \bar{w}}{d r}=-\frac{2 \tau_{w}}{D} \frac{r}{\mu\left(1+\mu_{T} / \mu\right)}
$$

and

$$
\frac{d \bar{h}}{d r}=\frac{4 c_{p} q_{w}}{D} \frac{\int_{0}^{r} \frac{\bar{w}}{\bar{w}_{b}} r d r}{\lambda\left(1+\frac{\operatorname{Pr}}{\operatorname{Pr}_{T}} \frac{\mu_{T}}{\mu}\right) r}
$$

involving the bulk velocity defined as

$$
\bar{w}_{b}=\frac{8}{D^{2}} \int_{0}^{D / 2} \bar{w} r d r
$$

The eddy viscosity required for the integration of (11) is presently modelled using a mixing-length ansatz

$$
\mu_{T}=-\rho l_{m}^{2} \frac{d \bar{w}}{d r}
$$

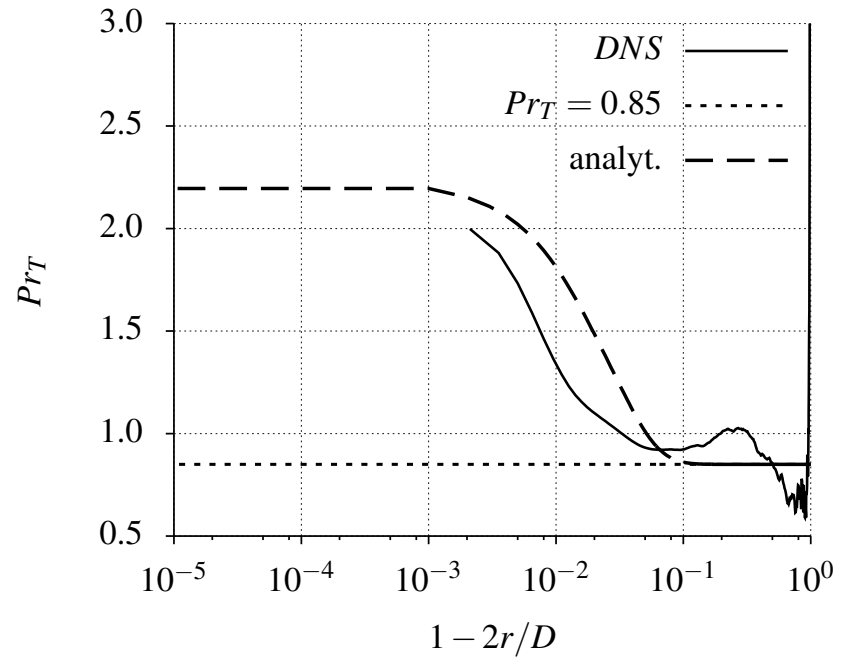

Figure 3. Radial variation of $\operatorname{Pr}_{T}$ predicted by the model and $D N S$ results for $R e_{\tau}=360, \operatorname{Pr}=20$

applying a van Driest-type dampening [10]. Rescaled with the wall friction velocity $w_{\tau}=\left(\tau_{w} / \rho\right)^{1 / 2}$ and the $\delta_{v}=\mu / \rho w_{\tau}$, the mixing length varies with the non-dimensional wall distance

$$
y^{+}=\left(\frac{1}{2}-\frac{r}{D}\right) \frac{\mu_{w}}{\mu} R_{\tau}
$$

as

$$
l_{m}^{+}=\frac{\rho w_{\tau} l_{m}}{\mu}=\kappa y^{+}\left[1-\exp \left(-\frac{y^{+}}{A^{+}}\right)\right]
$$

where the wall Reynolds number $R e_{\tau}=\rho w_{\tau} D / \mu_{w}$. It is important to note that the wall Reynolds number is modified here by the ratio $\mu_{w} / \mu$ to account for the effective increase/decrease of the locally relevant Reynolds number due to the change in the molecular viscosity depending on the temperature.

The radial variation of the enthalpy $\bar{h}(r)$ obtained from the integration of (12) can be definitely integrated, weighted with the mass flux $\rho \bar{w}$ over the whole cross-section, yielding the difference between the wall and the bulk enthalpies $\bar{h}_{w}-\bar{h}_{b}$. Recalling its definition dependent on the inverse of this difference

$$
N u_{w}=\frac{q_{w} D c_{p, b}}{\lambda_{w}\left(\bar{h}_{w}-\bar{h}_{b}\right)}
$$

the Nusselt number is obtained as

$$
\frac{1}{N u_{w}}=\frac{32}{D^{4}} \frac{c_{p, w}}{c_{p, b}} \int_{0}^{D / 2} \frac{\left(\int_{0}^{r}\left(\bar{w} / \bar{w}_{b}\right) r d r\right)^{2}}{\lambda / \lambda_{w} c_{p, w} / c_{p}\left(1+\operatorname{Pr}_{P} \operatorname{Pr}_{T} \mu_{T} / \mu\right) r} d r .
$$

The turbulent Prandtl number $\operatorname{Pr}_{T}$, which is required in this analytical solution, is modelled based on the radial variations of this parameter observed in the DNS results, as shown in Figure 2 . The proposed model variation assumes a generic radial profile which asymptotically approaches plateaus for wall distances $y^{+} \rightarrow 0$ and $y^{+} \rightarrow \infty$. The generic profile is mathematically prescribed as 


$$
\operatorname{Pr}_{T}=A+\frac{\operatorname{Pr}_{T, \infty}-A}{\left(1+\exp \left(-B y^{+}\right)\right)^{2}}
$$

where the model coefficients $A$ and $B$ basically determine the transition from the wall value towards the asymptotically reached bulk value $\operatorname{Pr}_{T, \infty}$ for $y^{+} \rightarrow \infty$. The model coefficients $A$ and $B$ need to be adapted dependent on the molecular Prandtl number, so that the trends observed in DNS are followed, and the Nusselt numbers predicted by the analytical expression (18) using this $\operatorname{Pr}_{T}$ model are in good agreement with experimental results. As seen from Figure 3, which exemplarily displays the predicted variation of $\operatorname{Pr}_{T}$ together with the corresponding $D N S$ data, the present model is capable to reproduce the salient features of the radial behavior of this important turbulence parameter very well.

\section{EXPERIMENTAL SETUP}

The experimental data used for calibration and validation of the present analytical model were acquired by measurements on a pipe flow test facility with a heating concept similar to that of [11]. The full experimental loop, as well as a detailed representation of the testsection, are shown in Figure 4. A typical thermal oil is used as the operating fluid with known relevant material properties. The fluid circulates in the loop, which essentially consists of a reservoir, a pump, a flowmeter, a heated testsection and a heat exchanger in order to ensure thermally stationary flow conditions. On its way through the testsection, the fluid passes first an unheated "entrance section", where the flow becomes dynamically fully developed. It continues flowing through a "heated testsection", where the fluid is heated by the constant wall heat flux $q_{w}$ to achieve thermally fully developed flow conditions. The wall material is electrically heated here by short circuiting the testsection with an electrical transformer providing high current at low voltage. Further downstream, the fluid further passes an unheated thermal equalization section, where

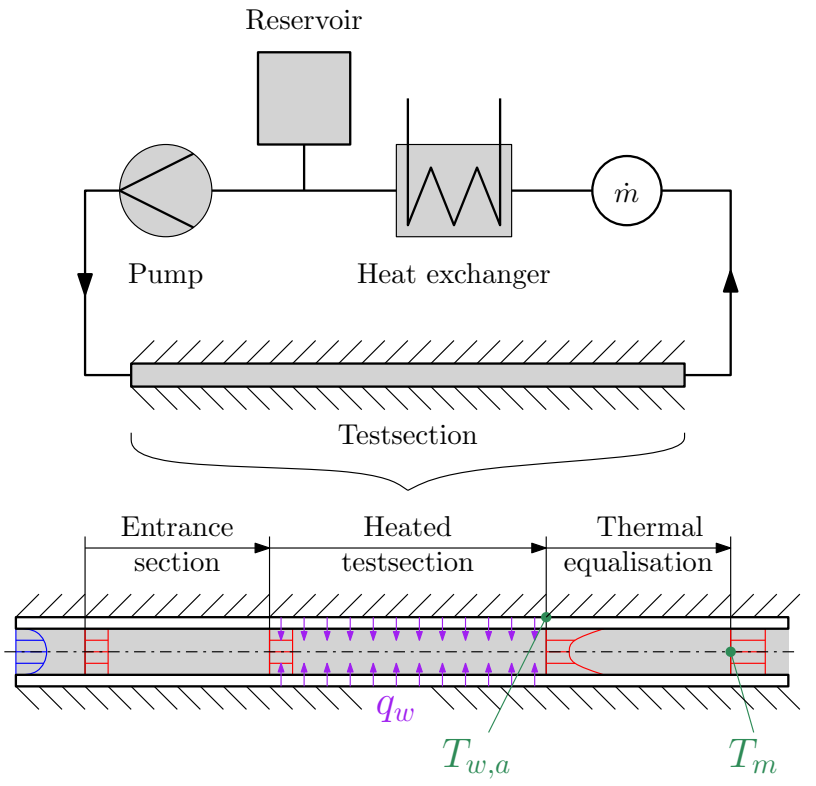

Figure 4. Schematic of the experimental facility
Table 1. Experimental cases

\begin{tabular}{ccccccc}
\hline$\#$ & $R e_{w}\left(R e_{\tau}\right)$ & $P r_{w}$ & $\dot{m}$ & $T_{m}$ & $T_{w}$ & $q_{w}$ \\
& - & - & $k g / s$ & $K$ & $K$ & $W / m^{2}$ \\
\hline M1 & $4800(335)$ & 21 & 0.046 & 428.0 & 474.4 & 24870 \\
M2 & $7290(500)$ & 24 & 0.080 & 460.7 & 460.7 & 31340 \\
M3 & $10960(710)$ & 27 & 0.143 & 445.4 & 445.4 & 30260 \\
\hline
\end{tabular}

the temperature can reach a uniform value over the crosssection, which represents the bulk temperature $T_{m}$. Both the wall temperature at the end of the heated section $T_{w}$, and the bulk temperature at the exit of the equalization section $T_{m}$ are measured using PT-100 sensors. The pipe is made of steel with $d_{i}=12 \mathrm{~mm}$ and $d_{o}=15 \mathrm{~mm}$ inner and outer diameters, respectively. The axial lengths of the "entrance section", "heated testsection" and "thermal equalization section" are $1.2 \mathrm{~m}, 2 \mathrm{~m}$ and $1.6 \mathrm{~m}$, respectively. The pressure drop is measured as well using pressure probes located at two axially distant positions. The Nusselt number is determined as

$$
N u_{w}=\frac{\alpha D}{\lambda_{w}}=\frac{q_{w} D}{\lambda_{w}\left(T_{w}-T_{m}\right)},
$$

where the heat flux can be computed based on the electrical input energy $P_{e l}=U I$. In order to avoid heat losses, the whole testsection is thermally isolated. The three experimental cases considered here are listed in Table 1. The bulk Reynolds numbers $R e_{w}=4 \dot{m} / D \mu_{w}$ are based on the mass flow rates $\dot{m}$ measured with the flowmeter, while the wall friction Reynolds number $R e_{\tau}=\rho w_{\tau} / \mu_{w}$ is obtained from the measured pressure drop.

\section{RESULTS AND DISCUSSION}

As seen from Table 1, the case M1 is to a certain extent comparable with the DNS case for $R e_{\tau}=360$ and $\operatorname{Pr}=20$, except for the influence of the temperature dependent material properties, which was not accounted for by the DNS. For the considered working fluid, the temperature most significantly changes the dynamic viscosity. This is clearly demonstrated by its strong radial variation shown in Figure 5 as produced by the analytically computed temperature profiles for M1, M2, and M3. The local viscosity sharply increases near the wall and reaches a level up to more than $60 \%$ higher than the reference value at the wall for the case M1. The comparison against the DNS results is still useful to demonstrate the possibly big influence of the temperature dependence of the material properties, especially of the dynamic viscosity, which is mostly neglected in DNS of turbulent flow with heat transfer. The DNS data are therefore included in the present discussion of the analytical results for the case M1. The analytically computed solution for the axial velocity always used a standard setting for the von Kármán constant and the walldampening parameter, $\kappa=0.40$ and $A^{+}=26$, respectively, as required for the mixing length specified by equation (16). Figure 6 shows the varation of this length obtained for M1, together with the mixing length reproduced from the DNS results. The analytical profile shows the right trend, with some considerable local 
Table 2. Model coefficients for the turbulent Prandtl number

\begin{tabular}{ccccc}
$\#$ & $R e_{w}\left(R e_{\tau}\right)$ & $P r_{w}$ & $A$ & $B$ \\
\hline M1 & $4800(335)$ & 21 & 2.7 & 0.5 \\
M2 & $7290(500)$ & 24 & 2.8 & 1.8 \\
M3 & $10960(710)$ & 27 & 3.0 & 2.9 \\
\hline
\end{tabular}

deviations though. The observed local discrepancy to an essentially linear behavior is however not unexpected in the light of the considered low Reynolds number. The thickness of the inertial sublayer, where the mixing-length can be assumed as linearly varying with the wall distance, is very large only for high Reynolds numbers.

Figure 7 shows the analytically obtained radial velocity distribution, again plotted together with the DNS results. The impact of the increasing viscosity towards the center observed in Figure 5 is clearly seen here. Due to the locally increased viscosity, the analytical solution yields evidently a lower axial velocity for the considered wall friction, which is imposed by the setting of $R e_{\tau}=360$. This reduction finally brings the analytically predicted bulk velocity $\bar{w}_{b}$ very close to the experimentally measured value, as compared to the DNS assuming constant viscosity. The analytical solution for the heat transfer underlines the great importance of an appropriate description of the near-wall behavior of the turbulent Prandtl number, which is needed for the integration of (12) for the specific enthalpy. Figure 8 shows the radial variation of $\operatorname{Pr}_{T}$ obtained from the presently proposed model formulation (6) for the three considered cases. Using the model coefficients listed in Table 2 basically moves the transition from the inner bulk value closer to the wall, approaching a higher level as the Reynolds number and the molecular Prandtl number become higher.

The improved accuracy provided by the shown model variations for $\mathrm{Pr}_{T}$ becomes evident in the analytical predictions for the

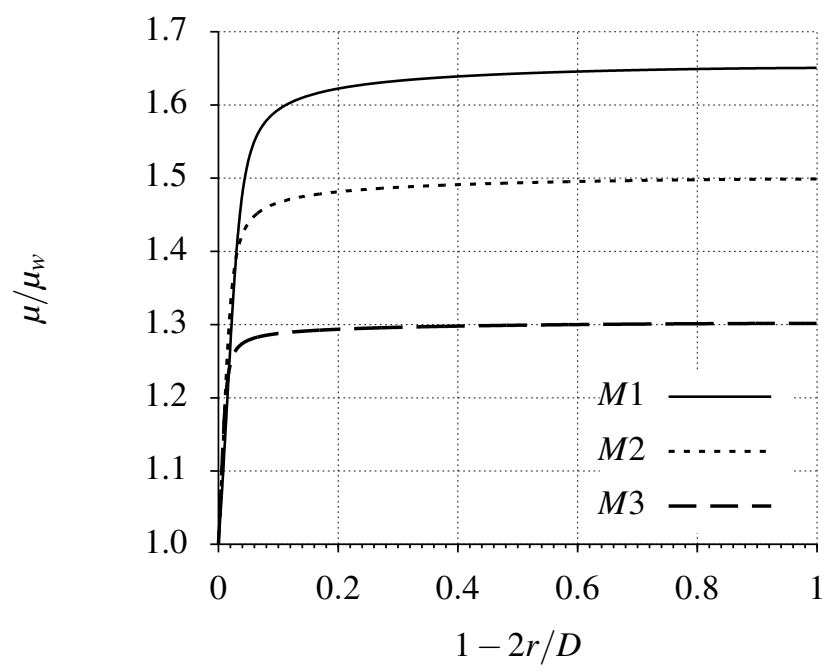

Figure 5. Radial distribution of $\mu / \mu_{w}$

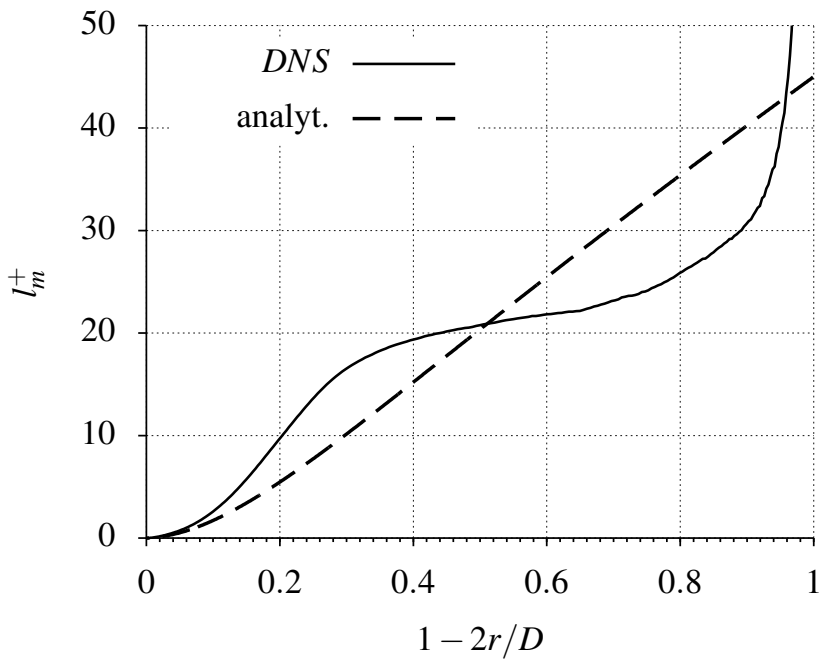

Figure 6. Radial variation of mixing-length for case M1

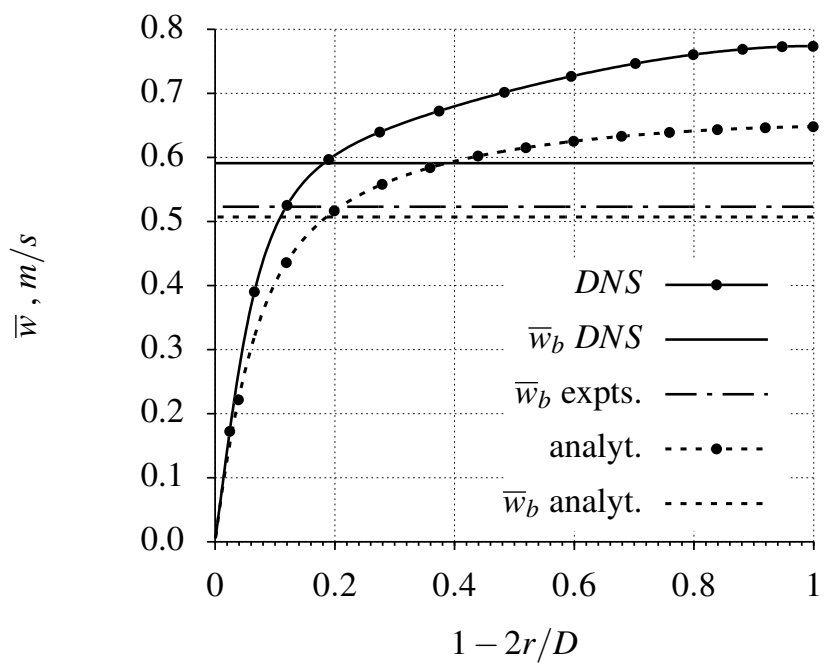

Figure 7. Radial variation of axial velocity for case M1

temperature. As shown in Figure 9 for case M1, using the model variations leads to notably reduced temperatures as compared to the analytical solution obtained with a uniform turbulent Prandtl number $\operatorname{Pr}_{T}=\operatorname{Pr}_{T, \infty}=0.85$. The resulting predicted lower level of the bulk temperature is in good agreement with the experiments. Again, the DNS results show a strong discrepancy to both analytical solutions due to the assumption of constant material properties.

The considerably accurate description delivered by the present analytical approach for the velocity and temperature translates into reliable predictions for global quantities derived therefrom, such as the mass flow rate and the Nusselt number. Tables 3 and 4 lists the analytically predicted mass flow rates and Nusselt numbers compared against the experimental data, respectively. Given the fact that the analytical model involves various simplifying assumptions, together with modelling uncertainties, the errors remain within an acceptable range. 


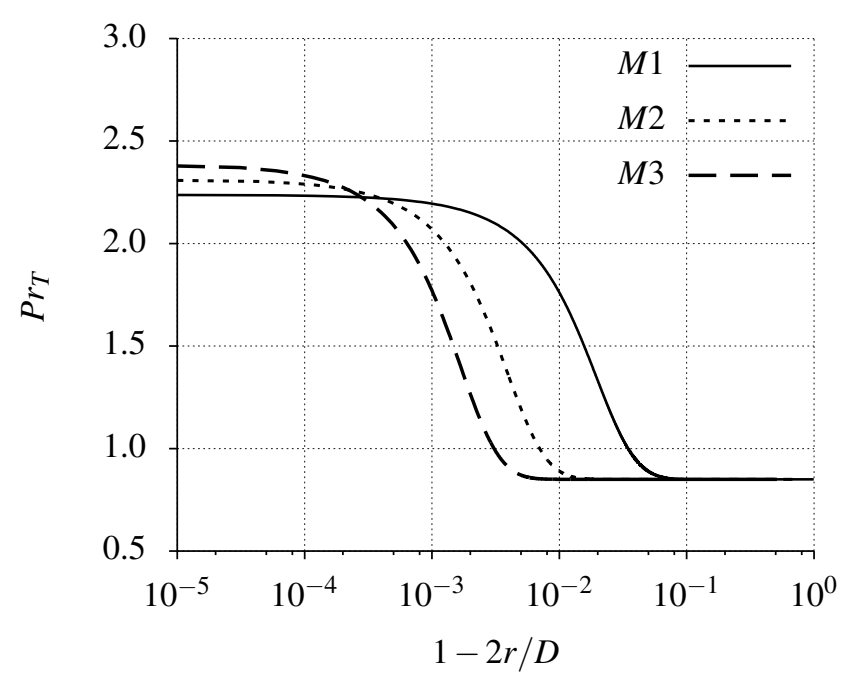

Figure 8. Model variation of $\operatorname{Pr}_{T}$ for all cases

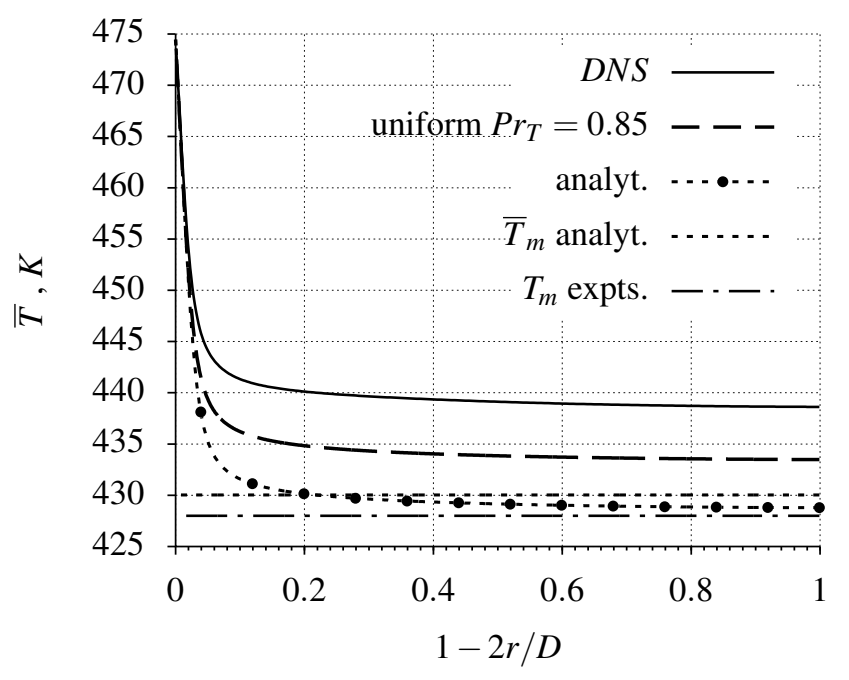

Figure 9. Radial variation of temperature for case M1

\section{CONCLUSIONS}

The presently proposed analytical approach is proven to provide a reliable description of turbulent flow with heat transfer at Prandtl numbers higher than unity. For the considered fully developped flow conditions, this concept represents an efficient alternative to computationally far more costly numerical approaches. The comparison against DNS data and experiments

Table 3. Mass flow rate

\begin{tabular}{ccccc}
\hline$\#$ & $\begin{array}{c}\operatorname{Re}_{w}\left(R e_{\tau}\right) \\
-(-)\end{array}$ & $\begin{array}{c}\text { Expts.: } \dot{m} \\
\mathrm{~kg} / \mathrm{s}\end{array}$ & $\begin{array}{c}\text { Analyt.: } \dot{m} \\
\mathrm{~kg} / \mathrm{s}\end{array}$ & $\begin{array}{c}\text { error } \\
\%\end{array}$ \\
\hline M1 & $4800(335)$ & 0.046 & 0.045 & -3.3 \\
M2 & $7290(500)$ & 0.080 & 0.077 & -3.4 \\
M3 & $10960(710)$ & 0.143 & 0.137 & -3.9 \\
\hline
\end{tabular}

Table 4. Nusselt number

\begin{tabular}{ccccc}
\hline$\#$ & $R e_{w}\left(R e_{\tau}\right)$ & Expts.: $N u_{w}$ & Analyt.: $N u_{w}$ & error $\%$ \\
\hline M1 & $4800(335)$ & 52.9 & 54.7 & 3.5 \\
M2 & $7290(500)$ & 94.4 & 90.1 & -4.5 \\
M3 & $10960(710)$ & 160.1 & 140.9 & -12.0 \\
\hline
\end{tabular}

highlights the significance of the dependence of the material properties on the temperature, as well as the importance of an appropriate modelling of the non-uniform behavior of the turbulent Prandtl number near the heated/cooled wall.

\section{ACKNOWLEDGMENTS}

The financial support from the Austrian Research Promotion Agency (FFG), the Virtual Vehicle competence center, and AVL List $\mathrm{GmbH}$ are gratefully acknowledged.

\section{REFERENCES}

[1] Hollingsworth, D. K., Kays, W. M., and Moffat, R. J., The measurement and prediction of heat transfer in a turbulent boundary layer in water, Proceedings of the 7th Symposium on Turbulent Shear Flows, Vol. 2, 1989, pp. 20.4.1-20.4.6.

[2] Blackwell, B. F., Kays, W. M., and Moffat, R. J., The turbulent boundary layer on a porous plate: an experimental study of the heat transfer behavior with adverse pressure gradients. Thermosciences Division, Dept. Mechanical Engineering, Stanford University, CA, NASA-Report No. HMT-16, 1972.

[3] Kozuka, M., Seki, Y., and Kawamura, H., DNS of turbulent heat transfer in a channel flow with a high spatial resolution. International Journal of Heat and Fluid Flow, Vol. 30, 2009, pp. 514524.

[4] Kang, C., and Yang, K. S., Effects of Schmidt number on nearwall turbulent mass transfer in pipe flow. Journal of Mechanical Science and Technology, Vol. 28, 2014, pp. 5027-5042.

[5] Cebeci, T., A model for eddy conductivity and turbulent Prandtl number. Journal of Heat Transfer, Vol. 95, 1973, pp. 227-234.

[6] Na, T.Y. and Habib, I.S., Heat transfer in turbulent pipe flow based on a new mixing length model, Applied Scientific Research, Vol. 28, 1973, pp. 302-314.

[7] Kays, W.M. and Crawford, M.E, Convective Heat and Mass transfer, McGraw-Hill Ryerson, 1980.

[8] Wassel, A. T., and Catton, I., Calculation of turbulent boundary layers over flat plates with different phenomenological theories of turbulence and variable turbulent Prandtl number. International Journal of Heat and Mass Transfer, Vol. 16, 1973, pp. 1547-1563.

[9] Petukhov, B.S., Heat transfer and friction in turbulent pipe flow with variable physical properties, Advances in Heat Transfer, Vol. 6, 1970, pp. 503-564.

[10] van Driest, E. R., On Turbulent Flow Near a Wall, Journal of Aeronautical Science, Vol. 23, 1956, pp. 1007-1011.

[11] Ghajar, Afshin J and Tam, Lap-Mou, Heat transfer measurements and correlations in the transition region for a circular tube with three different inlet configurations, Experimental Thermal and Fluid Science, Vol. 8, 1994, pp. 79-90. 